\title{
Generalized Synchronization of Different Dimensional Spatial Chaotic Dynamical Systems
}

\author{
Quan Hai ${ }^{1,2}$, Shutang Liu ${ }^{1, *}$, Changquan $\mathrm{Hu}^{1}$ \\ ${ }^{1}$ College of Control Science and Engineering, Shandong University, Jinan 250061, China \\ ${ }^{2}$ College of Mathematics Science, Inner Mongolia Normal University, Huhhot 010022, China
}

Copyright (c) 2016 by authors, all rights reserved. Authors agree that this article remains permanently open access under the terms of the Creative Commons Attribution License 4.0 International License

\begin{abstract}
In this paper, the generalized synchronization and the inverse generalized synchronization of different dimensional spatial chaotic dynamical systems are studied. The generalized synchronization results have been derived using active control method and Lyapunov stability theory. Numerical simulations are performed to verify the effectiveness of the proposed schemes.
\end{abstract}

Keywords Generalized Synchronization, Inverse Generalized Synchronization, Spatial Chaos, Lyapunov Stability

\section{Introduction}

In recent years, synchronization of chaotic systems has drawn much attention due to its wide application in various fields of physics and engineering $[1,2,3]$. The generalized synchronization of 1D chaos systems has been a topic of current focus $[4,5,6,7,8]$. For 2D spatial chaos, however, current research only focuses on the following spatially generalized Logistic system [9]

$$
x_{m+1, n}+\omega x_{m, n+1}=1-a\left((1+\omega) x_{m, n}\right)^{2},
$$

where $\omega$ is a real constant, $\mu$ is a positive parameter and $m, n \in N_{r}=\{r, r+1, r+2, \cdots \mid r$ is an integer and $r \leq 0\}$. The basic characteristics of system (1), such as the fixed plane, spatial Lyapunov exponents, spatial periodic orbit, stability of spatially periodic orbit, spatial chaos, spatial fractal, spatial chaos control and spatial chaotic synchronization, have obtained very rich contents $[10,11,12,13,14,15$, $16,17,18]$.

Based on Lyapunov stability theory, constructive schemes are presented in this paper to research the generalized synchronization and the inverse generalized synchronization between different dimensional spatial chaotic dynamical systems. Besides, the new synchronization criterions are established in the form of simple algebraic conditions which are very convenient to be verified.
The remainder of this paper is organized as follows: In Sect. 2, the generalized synchronization of spatial chaotic dynamical systems is investigated; In Sect. 3, the inverse generalized synchronization of spatial chaotic dynamical systems is studied; Sect. 4 demonstrates the proposed synchronization schemes and the derived theoretical results by using numerical examples and simulations, and Sect. 5 is the final conclusion.

\section{Generalized Synchronization of Spatial Chaotic Dynamical Systems}

Consider the following drive spatial chaotic system

$$
X_{m+1, n}+\omega X_{m, n+1}=f_{1}\left(\omega, X_{m, n}\right),
$$

where $X_{m, n}=\left(x_{m, n}^{1}, x_{m, n}^{2}, \cdots, x_{m, n}^{s}\right)^{T} \in \mathbb{R}^{s}$ is the state vector and $f_{1}: \mathbb{R}^{s} \rightarrow \mathbb{R}^{s}$ contains the linear and nonlinear parts of the drive system. As far as the response system is concerned, consider the following controlled chaotic system

$$
\begin{aligned}
Y_{m+1, n}+\omega Y_{m, n+1}= & B\left[(1+\omega) Y_{m, n}\right]+ \\
& g_{1}\left(\omega, Y_{m, n}\right)+U,
\end{aligned}
$$

where $Y_{m, n}=\left(y_{m, n}^{1}, y_{m, n}^{2}, \cdots, y_{m, n}^{t}\right)^{T} \in \mathbb{R}^{t}, B$ is an $m \times m$ matrix that represents the linear part of system dynamics, $g_{1}: \mathbb{R}^{t} \rightarrow \mathbb{R}^{t}$ is the nonlinear part of response system (3), and $U=\left(u_{i}\right)_{1 \leq i \leq t} \in \mathbb{R}^{t}$ is the vector controller to be determined.

Next, we introduce the definition of generalized synchronization for coupled spatial chaotic systems given in Eqs. (2) and (3).

Definition 1 The drive system (2) and the response system (3) are said to be generalized synchronization with respect to vector map $\Phi$ if there exists a controller $U=\left(u_{i}\right)_{1 \leq i \leq t} \in \mathbb{R}^{t}$ and a given map $\Phi: \mathbb{R}^{s} \rightarrow \mathbb{R}^{t}$ such that

$$
\lim _{\substack{m \rightarrow \infty \\ n \rightarrow \infty}}\left\|e_{m, n}\right\|=\lim _{\substack{m \rightarrow \infty \\ n \rightarrow \infty}}\left\|Y_{m, n}-\Phi\left(X_{m, n}\right)\right\|=0 .
$$


In order to study the generalized synchronization of the dynamical systems given in Eqs. (2) and (3), we discuss the asymptotic stability of the zero solutions of synchronization error system $e_{m, n}=Y_{m, n}-\Phi\left(X_{m, n}\right)$. We design controller $U$ such that the solution of error system $e_{m, n}^{i} \rightarrow 0, i=$ $1,2, \cdots, s$, as $m, n \rightarrow \infty$.

In this case, the error dynamics between drive system (2) and response system (3) can be derived as

$$
\begin{aligned}
& e_{m+1, n}+\omega e_{m, n+1} \\
& =B\left[(1+\omega) Y_{m, n}\right]+g_{1}\left(\omega, Y_{m, n}\right)- \\
& \quad \Phi\left(f_{1}\left(\omega, X_{m, n}\right)\right)+U .
\end{aligned}
$$

To achieve the generalized synchronization between systems (2) and (3), we can choose vector controller $U$ as follows

$$
\begin{aligned}
U= & -L_{1}\left[(1+\omega) Y_{m, n}\right]- \\
& g_{1}\left(\omega, Y_{m, n}\right)+\Phi\left(f_{1}\left(\omega, X_{m, n}\right)\right)+ \\
& \left(L_{1}-B\right) \Phi\left((1+\omega), X_{m, n}\right),
\end{aligned}
$$

where $L_{1} \in \mathbb{R}^{t \times t}$ is an unknown control matrix to be determined. By substituting Eq.(6) into eq.(5), the error system can be described as

$$
e_{m+1, n}+\omega e_{m, n+1}=\left(B-L_{1}\right)\left[(1+\omega) e_{m, n}\right] .
$$

Theorem 1 If we select control matrix $L_{1}$ such that $P_{1}=$ $I-\left(B-L_{1}\right)^{T}\left(B-L_{1}\right)$ is a positive definite matrix, then the drive system (2) and the response system (3) are globally generalized synchronization with respect to $\Phi$, under controller law (6).

Proof. Constructing the candidate Lyapunov function in the form

$$
V\left(\omega, e_{m, n}\right)=\left[(1+\omega) e_{m, n}\right]^{T}\left[(1+\omega) e_{m, n}\right] .
$$

The difference of $V\left(\omega, e_{m}, n\right)$ along the solution to (7) is

$$
\begin{aligned}
& \Delta V\left(\omega, e_{m, n}\right) \\
&=\left[e_{m+1, n}+\omega e_{m, n+1}\right]^{T}\left[e_{m+1, n}+\omega e_{m, n+1}\right]- \\
& {\left[(1+\omega) e_{m, n}\right]^{T}\left[(1+\omega) e_{m, n}\right] } \\
&= {\left[\left(B-L_{1}\right)(1+\omega) e_{m, n}\right]^{T}\left[\left(B-L_{1}\right)(1+\omega) e_{m, n}\right]-} \\
& {\left[(1+\omega) e_{m, n}\right]^{T}\left[(1+\omega) e_{m, n}\right] } \\
&=-\left[(1+\omega) e_{m, n}\right]^{T} P_{1}\left[(1+\omega) e_{m, n}\right] \\
&< 0 .
\end{aligned}
$$

Thus, from the Lyapunov stability theory, it is immediate that

$$
\lim _{\substack{m \rightarrow \infty \\ n \rightarrow \infty}} e_{m, n}^{i}=0, \quad(i=1,2, \cdots, t) .
$$

So, the zero solution of error system (7) is globally asymptotically stable, and therefore, systems (2) and (3) are globally generalized synchronization.

\section{Inverse Generalized Synchroniza- tion of Spatial Chaotic Dynamical Systems}

Now, we consider the drive and the response chaotic systems as,

$$
\begin{aligned}
X_{m+1, n}+\omega X_{m, n+1}= & A\left[(1+\omega) X_{m, n}\right]+ \\
& f_{2}\left(\omega, X_{m, n}\right), \\
Y_{m+1, n}+\omega Y_{m, n+1}= & g_{2}\left(\omega, Y_{m, n}\right)+U,
\end{aligned}
$$

where $X_{m, n}=\left(x_{m, n}^{1}, x_{m, n}^{2}, \cdots, x_{m, n}^{s}\right)^{T} \in \mathbb{R}^{s}$ and $Y_{m, n}=$ $\left(y_{m, n}^{1}, y_{m, n}^{2}, \cdots, y_{m, n}^{t}\right)^{T} \in \mathbb{R}^{t}$, are state vectors of the drive system and the response system, respectively, $A \in \mathbb{R}^{s \times s}$ is an $s \times s$ matrix that represents the linear part of system dynamics, $f_{2}: \mathbb{R}^{s} \rightarrow \mathbb{R}^{s}$ is the nonlinear part of drive system (11), $g_{1}: \mathbb{R}^{t} \rightarrow \mathbb{R}^{t}$ contains the linear and nonlinear parts of response system (12), and $U \in \mathbb{R}^{t}$ is the vector controller to be determined.

The definition of inverse generalized synchronization for coupled chaotic systems given in Eqs.(11) and (12) is given by

Definition 2 The drive system (11) and the response system (12) are said to be inverse generalized synchronization with respect to vector map $\Psi$ if there exists a controller $U=\left(u_{i}\right)_{1 \leq i \leq t} \in \mathbb{R}^{t}$ and a given map $\Psi: \mathbb{R}^{s} \rightarrow \mathbb{R}^{t}$ such that

$$
\lim _{\substack{m \rightarrow \infty \\ n \rightarrow \infty}}\left\|e_{m, n}\right\|=\lim _{\substack{m \rightarrow \infty \\ n \rightarrow \infty}}\left\|X_{m, n}-\Psi\left(Y_{m, n}\right)\right\|=0 .
$$

In order to study inverse generalized synchronization of the dynamical systems given in Eqs. (11) and (12), we discuss the asymptotic stability for the zero solutions of synchronization error system $e_{m, n}=Y_{m, n}-\Psi\left(X_{m, n}\right)$. We design controller $U$ such that the solution of error system $e_{m, n}^{i} \rightarrow 0, i=1,2, \cdots, s$, as $m, n \rightarrow \infty$.

In this case, the error dynamics between drive system (11) and response system (12) can be derived as

$$
\begin{aligned}
& e_{m+1, n}+\omega e_{m, n+1} \\
& =A\left[(1+\omega) X_{m, n}\right]+f_{2}\left(\omega, X_{m, n}\right)- \\
& \quad \Psi\left(g_{2}\left(\omega, Y_{m, n}\right)+U\right) .
\end{aligned}
$$

To achieve inverse generalized synchronization between systems (11) and (12), vector $U$ can be chosen as follows

$$
\begin{aligned}
U= & -g_{2}\left(\omega, Y_{m, n}\right)+ \\
& \Psi^{-1}\left[f_{2}\left(\omega, X_{m, n}\right)\right)+L_{2}(1+\omega) Y_{m, n}+ \\
& \left.\left(A-L_{2}\right) \Psi\left((1+\omega) X_{m, n}\right)\right],
\end{aligned}
$$

where $\Psi^{-1}: \mathbb{R}^{s} \rightarrow \mathbb{R}^{t}$ is the inverse of map $\Psi$ and $L_{2} \in$ $\mathbb{R}^{s \times s}$ is an unknown control matrix to be determined later. By substituting Eq.(15) into eq.(14), the error system can be described as

$$
e_{m+1, n}+\omega e_{m, n+1}=\left(A-L_{2}\right)\left[(1+\omega) e_{m, n}\right] .
$$


Theorem 2 If we select control matrix $L_{2}$ such that $P_{2}=$ $I-\left(A-L_{2}\right)^{T}\left(A-L_{2}\right)$ is a positive definite matrix, then the drive system (11) and the response system (12) are globally inverse generalized synchronization with respect to $\Psi$, under controller law (15).

Proof. Consider the candidate Lyapunov function in the following form

$$
V\left(\omega, e_{m, n}\right)=\left[(1+\omega) e_{m, n}\right]^{T}\left[(1+\omega) e_{m, n}\right] .
$$

The difference of $V\left(\omega, e_{m, n}\right)$ along the solution to (16) is

$$
\begin{aligned}
& \Delta V\left(\omega, e_{m, n}\right) \\
&=\left[e_{m+1, n}+\omega e_{m, n+1}\right]^{T}\left[e_{m+1, n}+\omega e_{m, n+1}\right]- \\
& {\left[(1+\omega) e_{m, n}\right]^{T}\left[(1+\omega) e_{m, n}\right] } \\
&= {\left[\left(A-L_{2}\right)(1+\omega) e_{m, n}\right]^{T}\left[\left(A-L_{2}\right)(1+\omega) e_{m, n}\right]-} \\
& {\left[(1+\omega) e_{m, n}\right]^{T}\left[(1+\omega) e_{m, n}\right] } \\
&=-\left[(1+\omega) e_{m, n}\right]^{T} P_{2}\left[(1+\omega) e_{m, n}\right] \\
&< 0 .
\end{aligned}
$$

Thus, we derive from the Lyapunov stability theory that

$$
\lim _{\substack{m \rightarrow \infty \\ n \rightarrow \infty}} e_{m, n}^{i}=0, \quad(i=1,2, \cdots, s) .
$$

So, the zero solution of the error system (16) is globally asymptotically stable, and therefore, systems(11) and (12) are globally inverse generalized synchronization.

\section{Simulation Examples}

In this section, two examples are provided to validate the proposed theoretical synchronization results.

\subsection{Example 1: Generalized Synchronization Between Spatial 2D Henon System and Spatial 2D Logistic System}

Here, we consider the spatial 2D Henon system as the drive system and the controlled spatial 2D logistic system as the response system. The spatial 2D Henon system can be described as

$$
\left\{\begin{aligned}
x_{m+1, n}^{1}+\omega x_{m, n+1}^{1}= & 1+b(1+\omega) x_{m, n}^{2}- \\
a & {\left[(1+\omega) x_{m, n}^{1}\right]^{2}, } \\
x_{m+1, n}^{2}+\omega x_{m, n+1}^{2}= & (1+\omega) x_{m, n}^{1},
\end{aligned}\right.
$$

where $\omega, b$ are real constants and $a$ is a positive parameter. Assuming that $a>1.55$, then spatial 2D Henon system (20) has a transversal homoclinic orbit for all $|b|<\varepsilon$ for some $\varepsilon>0$. In particular, for $|b|<\varepsilon$, system (20) is chaotic [19]. For example, when $(a, b, \omega)=(1.75,0.03,-0.02)$, system (20) is spatial chaos, as shown in Fig. 1.

The controlled spatial 2D Logistic system can be described as

$$
y_{m+1, n}+\omega y_{m, n+1}=1-\mu\left[(1+\omega) y_{m, n}\right]^{2}+U,
$$

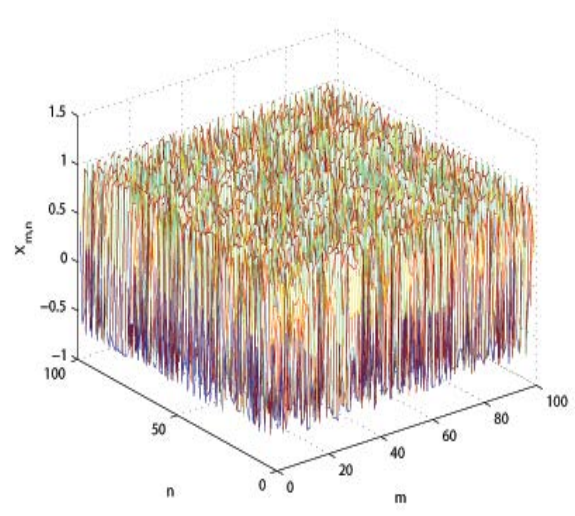

Figure 1. Chaotic behavior of system (20) with $\omega=-0.02, a=1.75$ and $b=0.03$

where $U$ is the scalar controller. Assuming that $\mu>1.55$, then, spatial 2D Logistic system (21) is chaotic in $\mathbb{R}^{2} \times \mathbb{R}$ in the sense of $\mathrm{Li}$ and Yorke [9]. For example, when $(a, \omega)=(1.68,-0.02)$, system (21) is spatial chaos, the spatial chaotic behavior of system (21) is shown in Fig. 2.

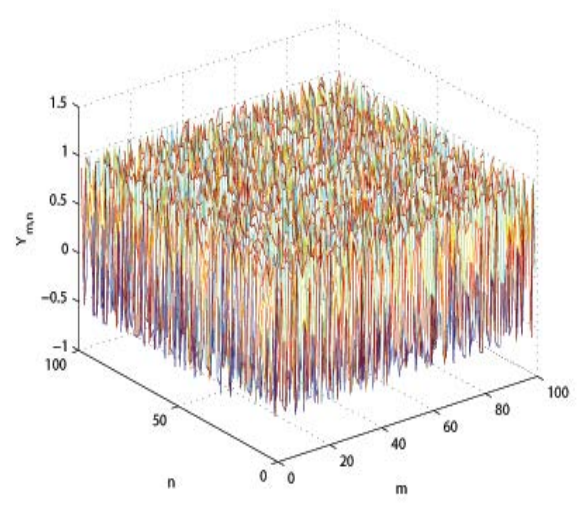

Figure 2. Chaotic behavior of system (21) with $\omega=-0.02$ and $a=1.68$.

Taking $(a, b, \omega)=(1.75,0.03,-0.02)$ and $(\mu, \omega)=$ $(1.68,-0.02)$, spatial 2D Henon system and spatial 2D Logistic system exhibit chaotic behavior. If we select map $\Phi$ : $\mathbb{R}^{2} \rightarrow \mathbb{R}$ as

$$
\Phi\left[\left(x_{m, n}^{1}, x_{m, n}^{2}\right)^{T}\right]=x_{m, n}^{1}+x_{m, n}^{2},
$$

and control matrix $L_{1}$ as

$$
L_{1}=0.72,
$$

then, according to our approach presented in Sect. 2, we obtain

$$
B=0,
$$

and

$$
g_{1}(Y(m, n))=1-\mu\left[(1+\omega) y_{m, n}\right]^{2} .
$$




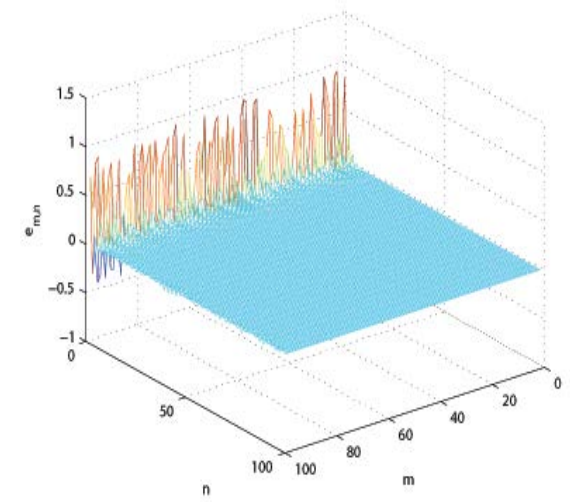

Figure 3. Time evolution of synchronization errors of systems (20) and (21).

By simple calculations, we can show that $I-\left(B-L_{1}\right)^{T}(B-$ $\left.L_{1}\right)$ is a positive definite matrix. Therefore, in this case, systems (20) and (21) are generalized synchronization. The error function evolution is shown in Fig. 3.

Specially, we take $\omega=0, n=0$, corresponding the spatial 2D Henon system (20) and the spatial 2D Logistic system (21) can be written as

$$
\left\{\begin{array}{l}
x_{m+1}^{1}=1+b x_{m}^{2}-a\left(x_{m}^{1}\right)^{2} \\
x_{m+1}^{2}=x_{m}^{1}
\end{array}\right.
$$

and

$$
y_{m+1}=1-\mu\left(y_{m}\right)^{2}+U .
$$

Clearly, when $(a, b)=(1.75,0.03)$ and $\mu=1.68$, systems (26) and (27) realize generalized synchronization as demonstrated in Fig. 4.

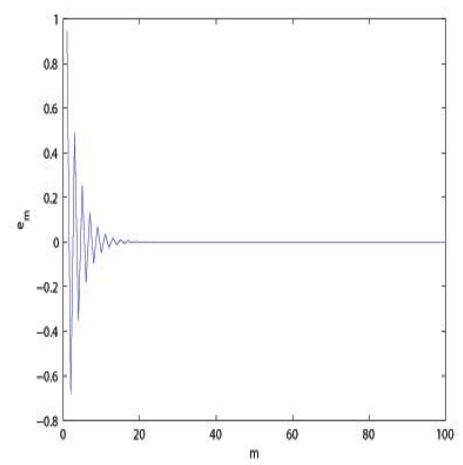

Figure 4. Time evolution of synchronization errors of systems (26) and (27).

\subsection{Example 2: Inverse Generalized Synchro- nization Between the Spatial 2D Henon System and the Spatial 2D Logistic System}

For systems (20) and (21), take $(a, b, \omega)=$ $(1.75,0.03,-0.02)$ and $(\mu, \omega)=(1.68,-0.02)$, the spatial 2D Henon system and the spatial 2D Logistic system exhibit chaotic behavior. If we select map $\Psi: \mathbb{R} \rightarrow \mathbb{R}^{2}$ as

$$
\Psi\left(y_{m, n}\right)=\left(y_{m, n}, 2 y_{m, n}\right)^{T},
$$

and control matrix $L_{2}$ as

$$
L_{2}=\left[\begin{array}{ll}
0.72 & b_{2}(1+\omega) \\
(1+\omega) & 0.22
\end{array}\right]
$$

then, according to our approach presented in Sect. 3, we obtain

$$
A=\left[\begin{array}{ll}
0 & b_{2}(1+\omega) \\
(1+\omega) & 0
\end{array}\right]
$$

and

$$
f_{2}(X(m, n))=\left[\begin{array}{l}
1-a\left[(1+\omega) x_{m, n}^{1}\right]^{2} \\
0
\end{array}\right] .
$$

By simple calculations, we can show that $I-\left(A-L_{2}\right)^{T}(A-$ $\left.L_{2}\right)$ is a positive definite matrix. Therefore, in this case, systems (20) and (21) are inverse generalized synchronization. Fig. 5 shows the error function evolution. On the hand, systems (26) and (27) realize inverse generalized synchronization as demonstrated in Fig. 6.

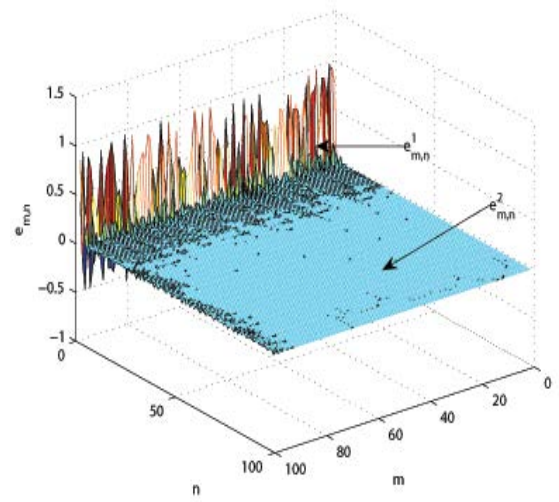

Figure 5. Time evolution of synchronization errors of systems (20) and (21).

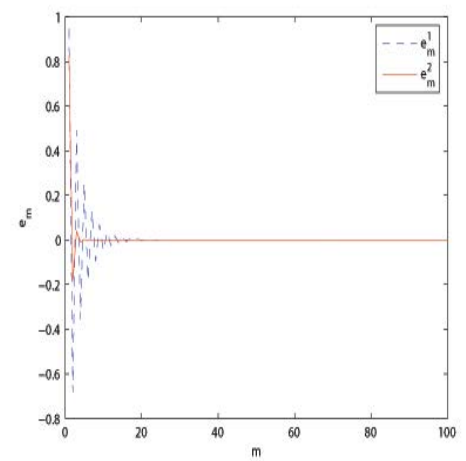

Figure 6. Time evolution of synchronization errors of systems (26) and (27). 


\section{Conclusions}

In this paper, the generalized synchronization and the inverse generalized synchronization of spatial chaotic dynamical systems are analyzed. What's more, a new control method is proposed in each case, and numerical simulations are performed to verify the effectiveness of the proposed schemes.

It should be pointed out that these analytic methods and theoretical results have in effect extended the generalized chaos synchronization from temporal systems to spatial systems, which is a significant development in the study on the subject of chaos synchronization.

\section{Acknowledgments}

Thank you for reviewer's valuable suggestions. The work was supported by the Key Program of National Natural Science Foundation of China (No. 61533011) and the National Nature Science Foundation of China (No. 61273088).

\section{REFERENCES}

[1] R. Roy, K. S. Thornburg. Experimental synchronization of chaotic lasers. Phys. Rev. Lett, 1994, 72: 2009-2012.

[2] G. S. Duane, P. J. Webster, J. B. Weiss. Go-occurrence of north and southern hemisphere blocks as partially synchronized chaos. J. Atoms. Sci., 1999, 56(24): 4183-4205.

[3] B. Blasius, L. Stone. Chaos and phase synchronization in ecological systems. Int. J. Bifur. Chaos, 10(10): 2361-2380.

[4] L. Junge, P. Ulrich. Synchronization using dynamic coupling. Phys. Rev. E, 2001, 64: 0552041-4.

[5] R. He, P. G. Vaidya. Analysis and synthesis of synchronous periodic and chaotic systems. Phys. Rev. E, 1992, 46(121): 7387-92.

[6] L. M. Pecora, T. L. Carroll. Synchronization in chaotic systems. Phys. Rev. Lett, 1990, 64(8): 821-4.
[7] U. Parlitz, L. Junge, L. Kocarev. Subharmonic entrainment of unstable period orbits and generalized synchronization. Phys. Rev. Lett, 1997, 79(17): 3158-61.

[8] F. R. Nikolai, M. S. Mikhail, S. T. Lev. Generalized synchronization of chaos in directionally coupled chaotic systems. Phys. Rev. E, 51(2): 980-94.

[9] S. T. Liu, G. Chen. On spatial Lyapunov exponents and spatial chaos. Int. J. Bifurc. Chaos, 2003, 13: 1163-1181.

[10] S. T. Liu, G. Chen. Asymptotic behavior of delay 2D discrete logistic systems. IEEE Trans. Circuits Syst. I Fundam. Theory Appl., 2002, 49: 1677-1682.

[11] S. T. Liu, G. Chen. Nonlinear feedback-controlled generalized synchronization of spatial chaos. Chaos Solitions Fractals, 2002, 22: 35-46.

[12] S. T. Liu, G. Chen. On spatial Lyapunov exponents and spatial chaos. Int. J. Bifurc. Chaos, 2003, 13: 1163-1181.

[13] G. Chen, S. T. Liu. On generalized synchronization of spatial chaos. Chaos Solitions Fractals, 2003, 15: 311-318.

[14] P. Wang, S. T. Liu. Feedback control and linear generalized synchronization of spatial-alternated Julia sets. Acta Phys. Sin., 2014, 63(6): 060503.

[15] D. Wang, S. T. Liu. Synchronization between the spatial Julia sets of complex Lorenz system and coplex Henon map. Nonlinear Dyn., 2015, 81: 1197-1205.

[16] P. Liu, C. A. Liu. Linear generalizad synchronization of spatial Julia sets. Int. J. Bifurc. Chaos, 2011, 21: 1281-1291.

[17] S. T. Liu, S. T. Wu. Uniformity of spatial physical motion systems and spatial chaos behavior in the sense of Li-York. Int. J. Bifurc. Chaos Appl. Sci. Eng., 2006, 16: 2697-2703.

[18] S. T. Liu, P. Liu. Spatial chaos on surface and its assoicated bifurction and Feigenbaum problem. Nonlinear Dyn., 2015, 81: 283-298.

[19] Q. Hai , S. T. Liu. Spatial dynamics behavior of spatial 2D Henon system. Complexity, 2016. (Accepted) 